\title{
Primary Prevention Strategies for Anthracycline Cardiotoxicity: A Brief Overview
}

\author{
Pierantonio Menna ${ }^{a}$ Emanuela Salvatorelli ${ }^{b}$

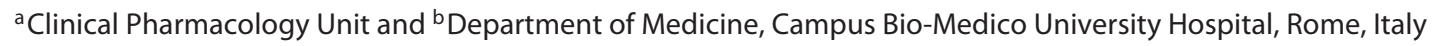

\section{Keywords}

Anthracyclines · Cardiotoxicity · Heart failure · Prevention

\begin{abstract}
The clinical use of doxorubicin and other antitumor anthracyclines is limited by a dose-related risk of cardiomyopathy and heart failure which may occur "on treatment" or any time, from months to years, after completing chemotherapy. Dose reductions diminish the incidence of cardiac events attributable to anthracyclines, but heart failure still occurs in some patients exposed to low or moderate anthracycline doses. Because anthracyclines improve the life expectancy of patients with, for example, breast cancer or lymphomas, preventing or diminishing the risk of early or delayed cardiotoxicity is of obvious clinical importance. Here, we briefly review some potential strategies of primary prevention that are based on what we know about the molecular mechanisms of cardiotoxicity, and what can be done, or might be done, to interfere with the pharmacokinetic, pharmacodynamic, and genetic determinants of cardiotoxicity.
\end{abstract}

(c) 2017 S. Karger AG, Basel

\section{KARGER}

(c) 2017 S. Karger AG, Basel

E-Mail karger@karger.com

www.karger.com/che

\section{Introduction}

Doxorubicin (DOX) and other antitumor anthracyclines, e.g., epirubicin (EPI), daunourubicin, and idarubicin, are widely used to treat many solid or hematologic malignancies; however, the clinical use of anthracyclines is limited by their severe cardiotoxicity, which correlates with the cumulative administered dose. In fact, anthracyclines induce cardiotoxicity that may progress toward dilated cardiomyopathy and systolic heart failure (HF). In the case of DOX, the risk of $\mathrm{HF}$ averages $5 \%$ at a cumulative dose of $400 \mathrm{mg} / \mathrm{m}^{2}$ but increases exponentially at higher doses [1]. Interestingly, however, studies of childhood and adult cancer survivors suggest that HF may occur also after cumulative doses $<400 \mathrm{mg} / \mathrm{m}^{2}$. Moreover, patients exposed to reportedly safe doses of anthracyclines may develop cardiomyopathy and HF any time after cancer diagnosis and treatment $[1,2]$. These findings suggest that there may be no safe dose of anthracyclines [3].

This having been recognized, clinicians should consider adequate measures of prevention for patients who are candidates for cumulative anthracycline doses $[1,4]$. Postchemotherapy surveillance is also needed to detect

Pierantonio Menna, $\mathrm{PhD}$

Clinical Pharmaology Unit, Campus Bio-Medico University Hospital Via Alvaro del Portillo, 200

IT-00128 Rome (Italy)

E-Mail p.menna@unicampus.it 
subclinical or mild asymptomatic cardiotoxicity before it progresses toward HF.

Here, we briefly review the potential strategies for preventing anthracycline cardiotoxicity.

\section{Primary Prevention of Anthracycline Cardiotoxicity}

\section{Pharmacokinetic Manipulations of Cardiac Exposure} to Anthracyclines

Anthracycline cardiotoxicity is a complex, multifactorial process that involves many potential molecular players [5]. Pharmacokinetic determinants of cardiotoxicity are relatively more obvious. For example, cardiotoxicity is reduced by replacing bolus administration with slow infusions over 24-96 h. This approach is based on an apparent dissociation between the pharmacokinetic determinants of anthracycline antiutumor activity versus cardiotoxicity. Whereas anthracycline activity correlates with total plasma exposure to anthracyclines, as exemplified by the area under the curve (AUC), cardiotoxicity correlates with the peak plasma level of anthracyclines $\left(\mathrm{C}_{\max }\right)$ and their penetration into cardiac tissue [6]. Substituting slow infusion for bolus administration does not significantly affect AUC, but it diminishes $\mathrm{C}_{\max }$ and anthracycline accumulation in the heart $[6,7]$. The activity and cardiac safety of slow anthracycline infusions have been documented in numerous studies. In some settings, slow infusions actually allowed for administering anthracyclines in cumulative doses otherwise known to induce HF [6]. The advantage of replacing bolus administration with slow infusion is nonetheless counterbalanced by the patient's discomfort due to prolonged hospitalization and the exacerbation of exposure effects such as myelotoxicity, mucositis, and alopecia. Moreover, slow infusions do not always prevent the risk of HF in survivors of childhood acute lymphoblastic leukemia [8]. Another possible concern originates from studies on breast cancer patients treated with DOX slow infusions. These patients developed an increased accumulation of DNA-oxidized bases in their peripheral blood mononuclear cells [9] This may have been the consequence of prolonged exposure to oxidative stress that is caused by the redox cycling of anthracyclines [6]. Should DNA damage also occur in cardiomyocytes and, more notably, in cardiac stem cells that are committed to repopulate the cardiac foci of anthracycline-induced damage, patients treated with anthracycline slow infusions would still carry a risk of developing HF [10]. Should DNA damage occur in hematopoietic precursors, this might also introduce an increased risk of secondary hematologic malignancy $[6,10]$.
The pharmacokinetic determinants of anthracycline cardiotoxicity are nonetheless liable to other manipulations and strategies for reducing cardiac exposure to anthracyclines. Cardiotoxicity can, in fact, also be reduced by replacing conventional anthracyclines with liposomeencapsulated ones. Two liposomal formulations have been approved for use with certain defined clinical indications. One liposomal DOX $\left(\right.$ Caelyx $^{\circledR}$ in Europe and Doxil $^{\circledR}$ in the USA) has polyethylene glycol embedded in the lipid layer; other formulations of DOX (Myocet ${ }^{\circledR}$ ) or daunorubicin (DaunoXome ${ }^{\circledR}$ ) adopt uncoated liposomes.

In brief, liposomal anthracycline formulations are too big to cross the gap junctions of endothelial linings in the heart and many other healthy tissues $[11,12]$. Steric hindrance limits anthracycline diffusion in extravascular compartments, which results in a higher $\mathrm{C}_{\max }$ and a lower distribution volume and clearance of liposomal formulations compared to conventional anthracyclines; however, the majority of circulating anthracycline remains embedded in the liposomal vesicle [13]. This reasoning anticipates that patients treated with liposomal anthracyclines would be exposed to very low levels of a circulating "free" drug, which eventually minimizes the risk due to cardiac exposure to freely diffusible anthracycline [6]. Liposomal formulations are nonetheless small enough to cross the very irregular and leaky microvasculature that characterizes solid tumors [6]. Following extravasation in tumors, liposomal formulations accumulate by virtue of processes that include the insufficient lymphatic drainage and increased interstitial pressure that also characterize many tumors. This is the so-called "enhanced permeability and retention effect" [13]. Moreover, the tumor microenvironment shows the potential for destabilizing the liposomal vesicle. This may occur through such diverse mechanisms as low $\mathrm{pH}$, the release of lipases from necrotic tumor cells, or the release of oxidizing agents from tumor-infiltrating inflammatory cells. Resident macrophages could contribute to the degradation of liposomal vesicles and release free anthracyclines, but the actual weight of this mechanism is uncertain [13, 14] (Fig. 1). Preclinical studies showed that liposomal formulations deliver substantial amounts of anthracycline in tumors while also delivering much smaller amounts of DOX in cardiac tissue compared to conventional DOX [13]. In some animal models, liposomal formulations actually expose tumor cells to higher amounts of free anthracycline than conventional formulations do [13]. For many reasons, liposomal anthracycline formulations might be considered prototypic examples of "targeted" drugs [10]. 
Many clinical studies show that liposomal anthracyclines are active and safe. A Cochrane analysis identified liposomal anthracyclines as the only formulations able to reduce the risk of anthracycline-related cardiotoxicity [15]. The efficacy and cardiac safety of liposomal DOX has been documented also under very unfavorable clinical conditions, e.g., when there is concomitant administration of DOX and the anti-Erbb2 monoclonal antibody, trastuzumab. Concomitant administration of conventional DOX and trastuzumab would precipitate serious cardiac events in women with Erbb2-overexpressing breast cancer, presumably because trastuzumab diminishes cardiac defense mechanisms against anthracycline injury [12]. In contrast, both uncoated and pegylated liposomal DOX could be safely administered with concomitant trastuzumab $[16,17]$, demonstrating that the limited cardiac penetration by the liposomal anthracycline prevented toxic synergism between trastuzumab and DOX.

Liposomal anthracyclines have been investigated in relatively few registration-oriented randomized trials. This means that liposomal formulations are approved for use only with limited clinical indications, e.g., metastatic breast cancer (uncoated liposomal DOX), metastatic breast cancer, advanced/refractory ovarian cancer, multiple myeloma, and AIDS-related Kaposi sarcoma (pegylated liposomal DOX), and AIDS-related sarcoma (liposomal daunorubicin) [1]. All other indications remain investigational, but clinicians should consider using liposomal anthracyclines, at least for patients who present at cancer diagnosis with cardiovascular risk factors (preexisting cardiac disease, arterial hypertension, diabetes, etc.) that are known to aggravate the risk of HF from conventional anthracyclines.

\section{Pharmacodynamic Approaches for Cardiac \\ Prevention}

For a long time, replacing a given anthracycline with an equiactive but less cardiotoxic analog has been considered the optimal and most obvious approach for preventing cardiotoxicity. Even though many anthracycline analogs have been tested preclinically and in early clinical studies, the search for a "less cardiotoxic" anthracycline has proven difficult, and unambiguous clinical validation of such an anthracycline is lacking. This probably reflects the multifactorial nature of anthracycline cardiotoxicity and the difficulties in disentangling the pharmacodynamic characteristics that eventually make one anthracycline less cardiotoxic than another.

Prevention of Anthracycline Cardiotoxicity

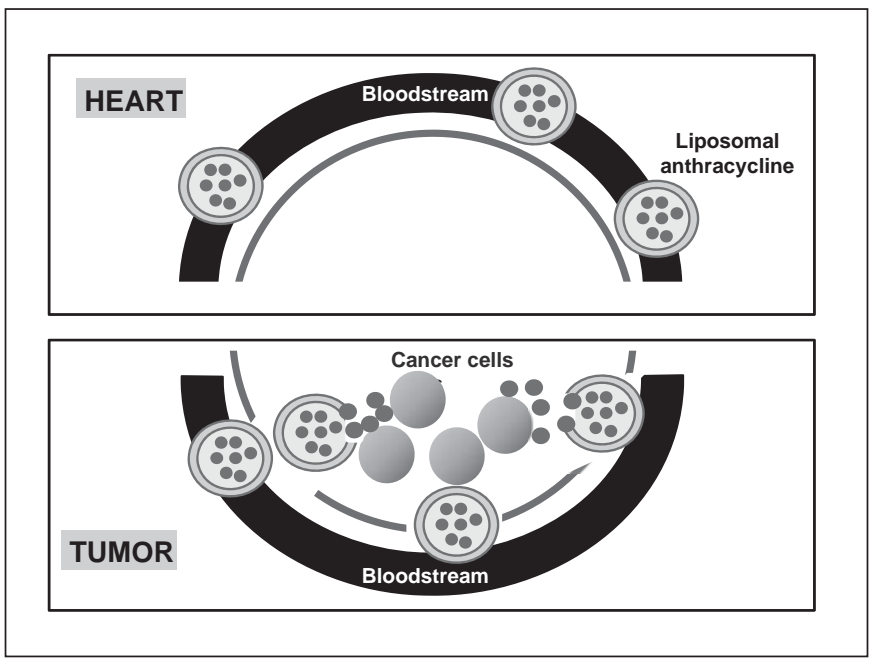

Fig. 1. The different pharmacokinetics of liposomal anthracyclines in tumors and the heart. The upper panel shows that sterical hindrance limits the diffusion of liposomal anthracyclines through the regular endothelial lining of the cardiac microvasculature. The bottom panel shows that liposomal formulations can diffuse through the irregular microvasculature of tumors and release free anthracycline in the interstitium.

DOX and other anthracyclines approved for clinical use share a tetracyclic quinone-hydroquinone moiety, an aminosugar (daunosamine), and a short side-chain with a carbonyl group. The quinone group undergoes a 1-electron reduction which forms a semiquinone radical. This quickly regenerates the parent quinone by reducing molecular oxygen to superoxide anion $\left(\mathrm{O}_{2}^{-}\right)$, and dismutation of the latter generates hydrogen peroxide $\left(\mathrm{H}_{2} \mathrm{O}_{2}\right)$. Thus, continued redox cycling of the quinone moiety exposes cardiomyocytes to reactive oxygen species (ROS) that, in concert with iron, may cause oxidative stress. To the extent that cardiomyocytes are relatively deficient in oxyradical-scavenging enzymes, such as superoxide dismutase and catalase, or glutathione peroxidase, the upregulation of oxidative stress might be highly deleterious to the heart $[18,19]$. In addition, the carbonyl group in the side-chain has been implicated to explain the mechanism or mechanisms of cardiotoxicity. In fact, 2-electron reduction of the carbonyl group generates a secondary alcohol metabolite that is significantly more polar than its parent anthracycline. It follows that, whereas anthracyclines are freely cleared from cardiomyocytes, anthracycline secondary alcohol metabolites show essentially no cardiac clearance and tend to form a long-lived toxic anthracycline reservoir [20]. Long-lived anthracycline metabolites contribute to inducing oxidative damage but can

Chemotherapy 2017;62:159-168 
Fig. 2. Metabolic determinants of anthracycline cardiotoxicity. One-electron redox cycling of the quinone moiety generates a semiquinone that converts oxygen to ROS. Further reactions of ROS with iron have been implicated to explain the development of cardiotoxicity. Two-electron reduction of the side-chain carbonyl group generates a secondary alcohol metabolite that, at odds with parent anthracyclines, shows a very limited cellular efflux and accumulates to form a long-lived anthracycline reservoir which is also implicated in cardiotoxicity. Modified from [12]. DOX, doxorubicin; DOXOL, doxorubicinol (secondary alcohol metabolite); ROS, reactive oxygen species.

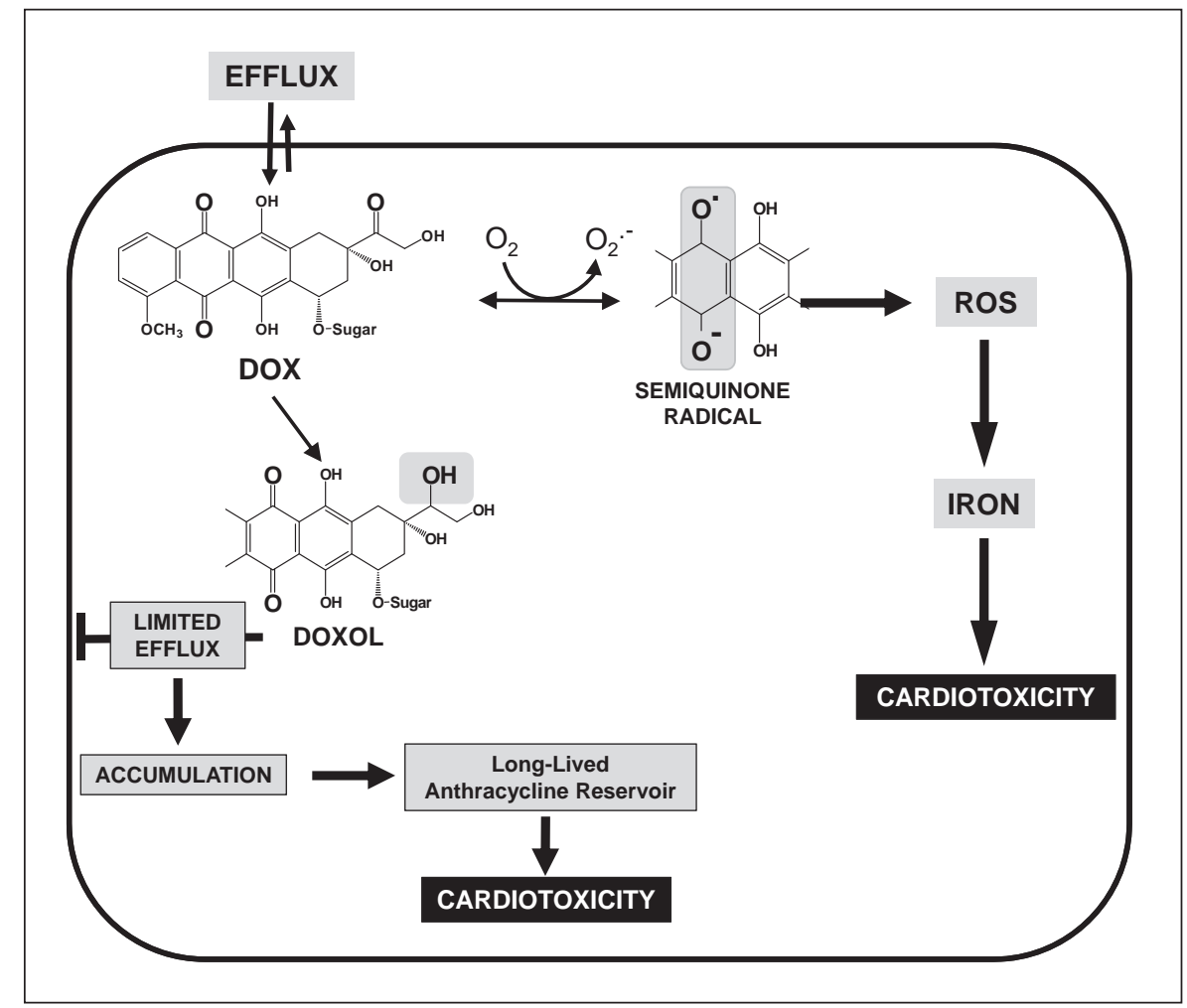

also cause oxidant stress-independent mechanisms of toxicity, e.g., the inhibition of numerous ATPases [21] or the activation of iron-regulatory proteins that control the cellular uptake and storage of iron [22,23] (Fig. 2).

The possible role of ROS and/or secondary alcohol metabolite in the pathogenesis of anthracycline-related cardiomyopathy and HF may or may not correlate with the clinical facts [6]. EPI, which differs from DOX by the epimerization of a hydroxyl group in daunosamine, shows a defective conversion to ROS and secondary alcohol metabolite [24]. And, not surprisingly, EPI is often said to be less cardiotoxic than DOX. Interestingly, however, the reduced cardiotoxicity of EPI versus DOX is only evident when they are given in equal amounts. Under such conditions, EPI shows a higher body clearance and a reduced antitumor activity. Should EPI be administered in higher doses to compensate for elimination and to attain the same activity as DOX, which is common practice in adjuvant therapy for breast cancer, the HF risk increases [25]. EPI would nonetheless retain advantages over DOX if the 2 were to be combined with tubulineactive poisons like taxanes, also common in adjuvant therapy of breast cancer. Taxanes, like paclitaxel and docetaxel, can in fact bind to a regulatory site of the re- ductases that convert anthracyclines to secondary alcohol metabolites, and, by doing so, they have been shown to increase DOX reduction to its metabolite, doxorubicinol. This does not occur with EPI, which, in principle, may result in an improved cardiac tolerability of EPI-taxane regimens compared to DOX-taxane regimens [26]. Amrubicin and the novel anthracenedione, pixantrone, also show a defective conversion to ROS and/or secondary alcohol metabolite $[27,28]$. Unfortunately, both of these drugs are approved for use only under limited conditions, and comprehensive clinical comparisons with DOX or other anthracyclines are limited [1]. Pixantrone was less cardiotoxic than DOX in a phase II study on patients with newly diagnosed non-Hodgkin lymphoma [29]. Pixantrone was also reasonably cardiac-tolerable in patients with refractory/relapsed non-Hodgkin lymphoma that had been previously treated with DOX [30]. Again, this might identify pixantrone as one of the long-sought "less cardiotoxic" anthracyclines. This having been said, it is worth noting that the mechanisms of antitumor activity of pixantrone are substantially different from those of DOX and other anthracenediones, which suggests that pixantrone should not be grouped with anthracyclinelike drugs $[31,32]$. 
Other strategies for reducing anthracycline cardiotoxicity have been based on the coadministration of drugs or natural compounds that fortify cardiomyocyte defenses against oxidative stress. Exploratory clinical trials that probed the protective efficacy of high-dose vitamin E or $\mathrm{N}$-acetylcysteine were uniformly disappointing $[33,34]$. In nononcologic patients, less robust interventions with vitamin $\mathrm{E}$ would be sufficient to prevent or attenuate the incidence of cardiac events that presumably involve oxidative stress, e.g., nonfatal myocardial infarction in patients with coronary atherosclerosis, arrhythmias, reinfarction after aortocoronary bypass, and fatal infarction in smokers [35]. How can these discrepancies be reconciled in a cohesive picture? In the case of anthracyclinerelated cardiotoxicity, one might speculate that antioxidants fail to reach critical levels in cell "sanctuaries" exposed to oxidative stress. Alternatively, continued redox cycling of the quinone moiety does expose cardiomyocytes to oxidative stress, but this is relatively unimportant for the occurrence of cardiotoxicity $[10,35]$.

The only drug that has proven protective in preclinical and clinical settings is dexrazoxane (DRZ). This is a bisketopiperazine which diffuses in cardiomyocytes, undergoes stepwise hydrolysis of its piperazine rings, and releases a diacid diamide (code-named ADR 925) that is similar to EDTA and chelates iron before it converts $\mathrm{O}_{2}^{-}$ and $\mathrm{H}_{2} \mathrm{O}_{2}$ into more potent hydroxyl radicals or equally reactive iron-oxygen complexes [35]. The myocardial uptake of DRZ is extraordinarily rapid and approaches its maximum level within $1 \mathrm{~min}$, which anticipates a rapid exposure of cardiomyocytes to ADR 529 [36]. This is particularly relevant for clinical conditions when DRZ is infused just prior to the anthracycline. This having been said, how is it that cardiotoxicity could be prevented by DRZ but not antioxidants? Both compounds should be cardioprotective if one assumes that iron-catalyzed oxidative stress was involved [35]. Such a discrepancy needs to be addressed by considering the broad spectrum of anthracycline targets in tumors and cardiac cells.

The antitumor activity of anthracyclines builds on the formation and stabilization of a reaction intermediate in which DNA strands are cut and covalently linked to tyrosine residues of topoisomerase IIa. This reaction negates the resealing of DNA and leads to the formation and accumulation of protein-capped DNA double-strand breaks. Topoisomerase II $\alpha$-mediated DNA damage is then followed by cell growth arrest in G1 and G2, and programmed cell death eventually occurs [6]. Whereas topoisomerase IIa is expressed primarily in tumor cells and normal proliferating cells, cardiomyocytes and other quiescent nor-

Prevention of Anthracycline Cardiotoxicity mal cells express constitutive levels of a $\beta$-isoform of topoisomerase II that is also inhibited by anthracyclines. Anthracycline inhibition of topoisomerase II $\beta$ causes DNA double-strand breaks and cardiomyocyte death [37]. Topoisomerase II $\beta$ therefore represents a previously unrecognized and probably a major target of anthracycline cardiotoxicity. DRZ can compete for the ATP-binding site of topoisomerase II $\beta$, and, by so doing, it forces topoisomerase II $\beta$ to assume a closed-clamp configuration that precludes the formation of anthracycline-DNA-topoisomerase II $\beta$ complexes [38]. DRZ therefore prevents DNA damage and cardiomyocyte death [37, 38] (Fig. 3).

How can these notions help to reconcile the lack of protection by antioxidants with the efficient protection provided by DRZ? The main conclusion to be drawn is that iron chelation and the mitigation of oxidative stress might not represent the only or prevailing mechanism of cardioprotection by DRZ. Accordingly, it was found that there was marginal protection or lack of effect when anthracycline-treated animals were exposed to DRZ analogs that chelated iron but did not inhibit topoisomerases [39]. Likewise, new and more potent compounds, designed to intensify iron chelation and redox-inactivation, have failed to provide equal or better cardiac protection than DRZ [40, 41].

DRZ has prevented anthracycline-related cardiotoxicity in many clinical studies of both childhood and adult cancer patients, often allowing for anthracyclines to be administered in cumulative doses known to induce HF [42]. DRZ is the only drug to be approved by the US FDA for use as a cardiac protectant in patients exposed to anthracyclines. Regrettably, however, the clinical use of DRZ is limited by just 1 report of its possible interference with anthracycline activity in metastatic breast cancer [43]. An interference of DRZ with anthracycline activity, possibly mediated by DRZ binding to topoisomerase IIa [44], might, at least in principle, justify concerns about its negative influence on tumor response, but a plethora of studies now demonstrates that such interference does not occur or is clinically unimportant [45]. In spite of such compelling evidence, the American Society of Clinical Oncology, Chemotherapy, and Radiotherapy Expert Panel maintains a cautionary position and recommends using DRZ only under very limited conditions (e.g.) patients who have received $>300 \mathrm{mg} / \mathrm{m}^{2}$ of DOX for metastatic breast cancer and who may benefit from continued anthracycline treatment) [46].

The clinical use of DRZ has also been limited by concerns about an increased risk of second malignancies [47]. This was observed in survivors of young-adolescent Hodgkin lymphoma who had been treated with DOX in com- 


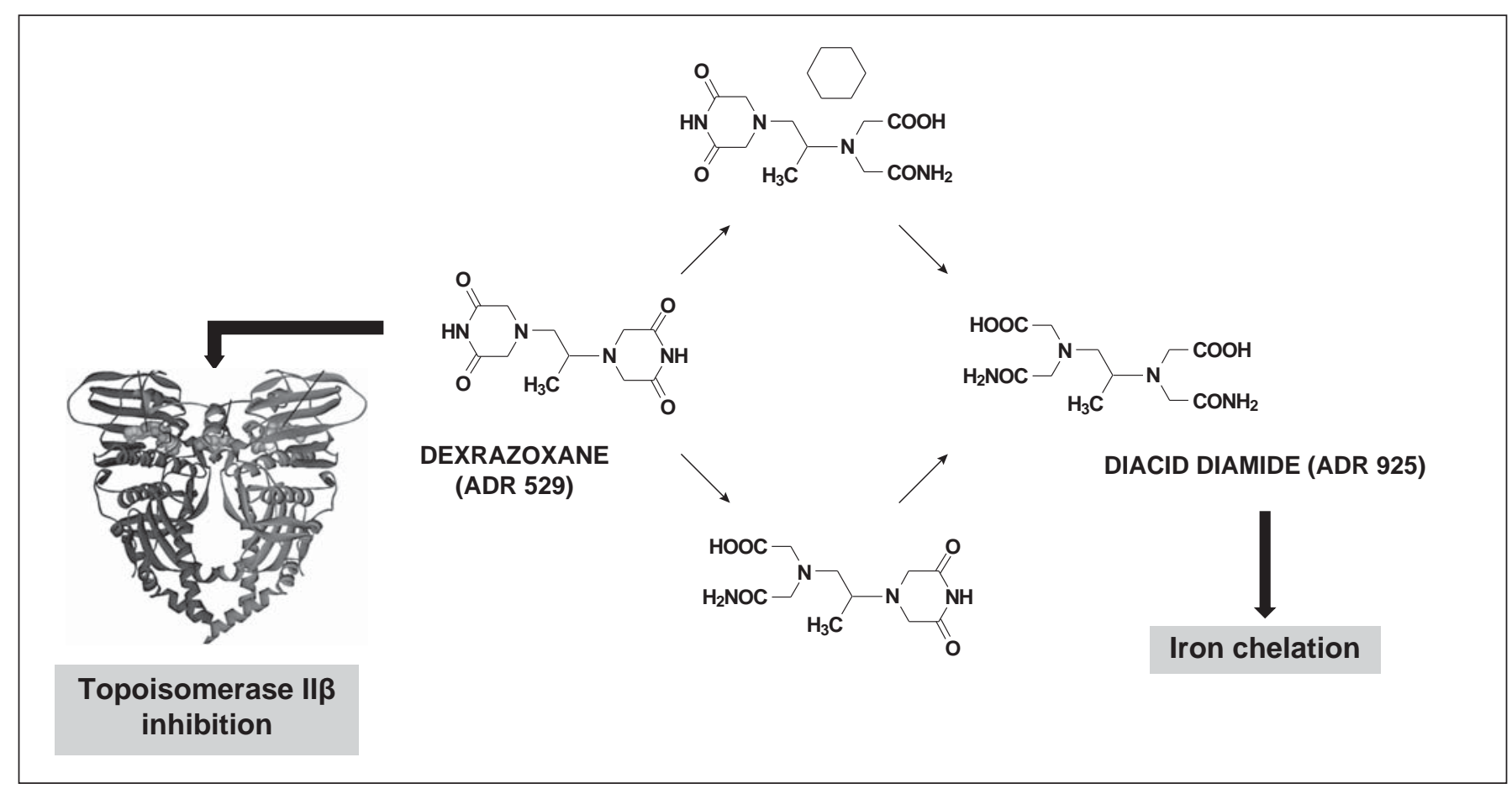

Fig. 3. Dual mode of action of dexrazoxane: it may prevent cardiotoxicity by inhibiting topoisomerase II $\beta$, which is constitutively expressed in cardiomyocytes, or by chelating iron through its diacid diamide product of hydrolysis, ADR 529.

bination with etoposide. Such concerns need to be interpreted by considering that etoposide is also a general topoisomerase inhibitor, and so patients exposed to DOXetoposide-DRZ combinations were actually receiving a cocktail of 3 topoisomerase inhibitors. Genetic instability may well have featured in these patients [10,47]. It was because of such concerns that the European Medicines Agency released a formal warning to contraindicate DRZ for use in children. However, as was the case about concerns about interference with antitumor activity, claims about an increased risk of secondary malignancy have been mitigated in several reports [48-50]. Risk-benefit analysis and pharmacological reasoning therefore support an unrestricted clinical use of DRZ in children and adolescents, with the only exception being those few patients who are candidates for etoposide-anthracycline combinations [51]. It is hoped that the European Medicines Agency will soon revise its position against using DRZ in children.

\section{Pharmacogenetic Prevention}

Some patients show a predisposition to develop HF. Predisposition means that cardiomyopathy progresses and HF develops even if patients were given cumulative anthracycline doses that were thought to be safe. In the case of DOX, this would be the case for patients treated with a maximum of $240-300 \mathrm{mg} / \mathrm{m}^{2}$ (vs. a $5 \%$ risk dose that is currently approximated to $400 \mathrm{mg} / \mathrm{m}^{2}$ ) [1]. Predisposition also means that cardiotoxicity may surface regardless of whether the patients presented at treatment with cardiovascular risk $[52,53]$.

It is now evident that many genetic modifiers can accelerate the course of development of anthracycline cardiotoxicity. One of the best known examples pertains to anthracycline secondary alcohol metabolites. In preclinical studies, mice with cardiac-specific overexpression of type 3 carbonyl reductase (CBR) exhibited an increased conversion of DOX to its secondary alcohol metabolite and an accelerated course of development of cardiomyopathy [54]. Conversely, mice with genetic deletion of type 1 CBR (CBR1) formed smaller amounts of alcohol metabolite and showed a reduced cardiotoxicity [55]. In clinical settings, a retrospective study of childhood cancer survivors showed that homozygosis for the gain-of-function CBR3 V244MG allele increased the risk of developing HF after low-to-moderate doses of anthracyclines [56]. Moreover, elevated expression of 
CBR1 may well explain the higher-than-expected rates of HF that occur in individuals with Down syndrome $[57,58]$.

Anthracycline conversion to secondary alcohol metabolites can be mediated by other enzymes as well. Inhibitor studies would in fact suggest that aldehyde reductases also contribute to generating anthracycline secondary alcohol metabolites in the human heart [59], but information about gain-of-function genetic variants of these distinct reductases is scant at this point in time.

Individual predisposition to anthracycline cardiotoxicity can also build on high expression levels of topoisomerase II $\beta$ [60] or genetic variants of topoisomerase II $\beta$ coregulating factors (retinoic acid receptor- $\gamma$ ) [61], polymorphisms of drug transporters that contribute to anthracycline distribution and elimination (ATP-binding cassette proteins of the multidrug resistance proteins family) [62], prooxidant enzymes (NADPH oxidase) [63], and matrix-remodeling enzymes (type 3 hyaluronan synthase) [64]. In brief, a constellation of factors may cause an unpredictable aggravation of cardiotoxicity.

Can genotyping be considered a novel strategy of primary prevention? The answer is positive on conceptual grounds but negative on practical grounds. Given the multifactorial nature of cardiotoxicity, each patient should be genotyped for $\geq 5$ potential predisposing factors. Screening for just 1 or 2 candidate genes would expose patients and doctors to false-negative information. On the other hand, recommendations about avoiding anthracyclines or reducing anthracycline dosages in patients with genetic predisposition should be supported by large prospective studies, which are not available at this point in time. This is an area of investigation that clearly needs to be developed.

\section{Cardiovascular Prophylaxis}

Primary prevention of anthracycline cardiotoxicity should also involve common sense procedures that apply to any patient at risk for cardiac events. Preexisting comorbidities (hypertension, systolic dysfunction, or metabolic disorders) or unfavorable lifestyle choices (smoking, overweight, or reduced physical activity) should be rigorously corrected as they have long been known to increase the risk of cardiotoxicity in patients exposed to anthracyclines [6]. Given that cardiotoxicity may occur any time after treatment with anthracyclines, and having emphasized that many genetic modifiers may increase the risk of developing HF, one should also ask whether cardiovascular prophylaxis is to be recommended also for patients without risk factors. This is a matter of debate as many doctors believe that, in low-risk patients, discomfort from antitumor drugs (nausea, vomiting, and asthenia) should not be compounded by discomfort from cardiovascular drugs (bradycardia, hypotension, fluid retention, and cough) [20]. Here, we first describe the available evidence for primary prevention with cardiovascular drugs, and then the risk versus benefit of adopting this measure.

Significant cardiac protection, measured as preservation or a limited decrease in left-ventricle ejection fraction (LVEF), was seen in studies of carvedilol $\left(\alpha_{1}\right.$ and $\beta_{1-2}$ adrenoceptor blocker) [65], nebivolol ( $\beta_{1}$ blocker) [66] or carvedilol in combination with the angiotensinconverting enzyme inhibitor, enalapril [67]. A reduced degree of protection was documented with metoprolol ( $\beta_{1}$ blocker) or enalapril alone [68]. In a recent study, the angiotensin II receptor blocker, candesartan, prevented the decline of LVEF in breast cancer patients receiving adjuvant anthracycline with or without subsequent trastuzumab. Metoprolol was ineffective in these patients [69]. Are these studies persuasive enough to recommend cardiovascular prophylaxis in any patient candidate for anthracyclines? As was mentioned for genotyping, this is one of those cases when the pros and cons need to be discussed on conceptual and practical grounds. We suggest that, conceptually speaking, there is no obvious obstacle to medicating patients candidate for anthracyclines, as the risk of HF is high enough to recommend that all cautionary measures be put into action. On practical grounds, protection with inhibitors of the renin-angiotensin system is feasible, cost-convenient, and very well characterized in terms of safety and compliance. One should have reservations if one looks at the robustness of the available studies, however. These were of a limited sample size and the protocol-specified end points often focused on marginal decrements of LVEF (e.g., $\geq 5 \%$ on MRI) [69]. Moreover, the available studies had no long-term follow-up, and so we do not actually know if primary prevention extends its beneficial effects in long-term survivors, who carry an increased risk of HF. When examined in a broader perspective, these reservations also raise questions as to whether primary prevention should be confined to a limited time interval or prolonged indefinitely. Having acknowledged that anthracyclines accumulate in cardiac tissue and prime patients to a lifetime risk of HF, we would suggest that pharmacologic cardioprotection should also be extended over time.

Other drugs merit consideration in the setting of primary prevention. An observational clinical cohort study of breast cancer patients treated with anthracyclines suggested that statins could also reduce the risk of HF [70]. This find- 
Table 1. Primary prevention strategies for anthracycline cardiotoxicity

\begin{tabular}{|c|c|c|c|}
\hline Strategy & Mechanism of protection & Clinical benefit & Disadvantages/limitations \\
\hline \multicolumn{4}{|c|}{ Pharmacokinetic manipulation of cardiac exposure } \\
\hline Slow infusions & lowered $\mathrm{C}_{\max }$ & $\begin{array}{l}\text { preservation of anthracycline } \\
\text { activity, with reduced } \\
\text { cardiac exposure to and } \\
\text { penetration by anthracyclines }\end{array}$ & $\begin{array}{l}\text { the patient undergoes prolonged } \\
\text { hospitalization and discomfort from } \\
\text { prolonged exposure; a lack of long-term } \\
\text { cardiac protection in some pediatric } \\
\text { settings; a reported accumulation of } \\
\text { DNA-oxidized bases in normal cells } \\
\text { (the effect of prolonged exposure to } \\
\text { ROS?) }\end{array}$ \\
\hline Liposomal formulations & $\begin{array}{l}\text { limited diffusion through the } \\
\text { endothelial lining of the cardiac } \\
\text { microvasculature }\end{array}$ & $\begin{array}{l}\text { preservation of antitumor } \\
\text { activity and improved cardiac } \\
\text { tolerability }\end{array}$ & limited approved indications \\
\hline \multicolumn{4}{|l|}{ Pharmacodynamic approaches } \\
\hline Antioxidants & $\begin{array}{l}\text { the mitigation of ROS-mediated } \\
\text { damage }\end{array}$ & $\begin{array}{l}\text { a reduced risk of cardiac } \\
\text { events }\end{array}$ & unproven efficacy \\
\hline Dexrazoxane & $\begin{array}{l}\text { iron chelation and mitigation of } \\
\text { ROS-mediated damage; inhibition of } \\
\text { topoisomerase II } \beta\end{array}$ & $\begin{array}{l}\text { prevention of cardiotoxicity } \\
\text { in both child and adult cancer } \\
\text { patients }\end{array}$ & $\begin{array}{l}\text { interference with anthracycline activity } \\
\text { and an increased incidence of second } \\
\text { malignancies (largely disproven) }\end{array}$ \\
\hline Less cardiotoxic analogs & $\begin{array}{l}\text { reduced ROS and alcohol } \\
\text { metabolite formation }\end{array}$ & a reduced incidence of $\mathrm{HF}$ & not definitely proven \\
\hline \multicolumn{4}{|l|}{ Pharmacogenetic prevention } \\
\hline $\begin{array}{l}\text { Screening for topoisomerase II } \beta \\
\text { levels and polymorphisms of CBR3, } \\
\text { coregulators of topoisomerase II } \beta \\
\text { expression, drug transporters, } \\
\text { NADPH oxidase, and } \\
\text { matrix-remodeling enzymes }\end{array}$ & $\begin{array}{l}\text { identification of patients at risk for } \\
\text { altered anthracycline distribution, } \\
\text { drug activation, oxidative stress, } \\
\text { DNA damage, and tissue repair }\end{array}$ & $\begin{array}{l}\text { the possibility for dose } \\
\text { adjustments and/or } \\
\text { replacing anthracyclines with } \\
\text { other drugs }\end{array}$ & $\begin{array}{l}\text { iovascular } \\
\text { investigational; a need for the screening } \\
\text { of multiple candidate genes; a lack of } \\
\text { prospective clinical trials }\end{array}$ \\
\hline \multicolumn{4}{|l|}{ Cardiovascular prophylaxis } \\
\hline Correction of comorbidities & reduced cardiac stress & a reduced incidence of $\mathrm{HF}$ & none \\
\hline $\begin{array}{l}\text { Coadministration of } \\
\text { cardiovascular drugs }\end{array}$ & reduced cardiac stress & a reduced incidence of $\mathrm{HF}$ & $\begin{array}{l}\text { limited trial evidence (but conceptually } \\
\text { recommended); a need for dose-finding } \\
\text { studies; the efficacy of long-term } \\
\text { administration is not yet known }\end{array}$ \\
\hline
\end{tabular}

ing appears consistent with the hitherto known cardioprotective effects of statins, such as the activation of nitric oxide synthase [71] and the opening of mitochondrial ATP-sensitive potassium channels [72]. The protective effects of statins are nonetheless most evident in patients who receive other cardiovascular drugs for preexisting risk factors. This raises questions as to whether the effects attributed to statins are actually due to the concomitant drugs or reflect the pleiotropic cardioprotective effects of statins in isolation, or in concert, with, for example, $\beta$ blockers or inhibitors of the renin-angiotensin system.

One last point for consideration pertains to the dosage of cardiovascular drugs. We do not know whether drugs should be given in "prophylactic" doses tailored to the characteristics of cancer patients and to the cumulative anthracycline dose, or in "therapeutic" doses similar to those rec- ommended for the general population. Prospective randomized trials are needed to define the efficacy and dose relations of primary prevention with cardiovascular drugs. Main strategies for primary prevention of anthracycline cardiotoxicity are summerized in Table 1.

\section{Conclusion}

Modern oncologists and hematologists know how to use anthracyclines. The cumulative doses used in the past are now replaced by lower cumulative doses that retain activity but have a lower risk of HF. Dose reductions actually represent the first and most obvious strategy of primary prevention. We have discussed how HF may nonetheless still occur in both low- and high-risk patients 
treated with these "safer" cumulative anthracycline doses. We call attention to opportunities for primary prevention that protect the heart before it develops clinically evident damage. Secondary prevention, guided by the early symptoms or laboratory findings of cardiomyopathy (e.g., echocardiographic abnormalities or increased levels of circulating biomarkers like troponin or natriuretic peptides), would still be appropriate, but is conceptually less effective. Some potential measures for primary prevention, like liposomal anthracycline or DRZ administration, should be given greater consideration.

\section{Disclosure Statement}

We have no conflicts of interest to declare.

\section{References}

1 Salvatorelli E, Menna P, Cantalupo E, Chello M, Covino E, Wolf FI, Minotti G: The concomitant management of cancer therapy and cardiac therapy. Biochem Biophys Acta 2015 1848:2727-2737

2 Menna P, Gonzalez Paz O, Chello M, Covino E, Salvatorelli E, Minotti G: Anthracycline cardiotoxicity. Expert Opin Drug Saf 2012; 11:S21-36.

3 Barry E, Alvarez JA, Scully RE, et al: Anthracycline-induced cardiotoxicity: course, pathophysiology, prevention and management. Expert Opin Pharmacother 2007;8:1039-1058.

4 Salvatorelli E, Menna P, Minotti G: Managing anthracycline-induced cardiotoxicity: beginning with the end in mind. Future Cardiol 2015;11:363-366.

5 Gianni L, Herman EH, Lipshultz SE, et al: Anthracycline cardiotoxicity: from bench to bedside. J Clin Oncol 2008;26:3777-3784.

6 Minotti G, Menna P, Salvatorelli E, et al: Anthracyclines: molecular advances and pharmacologic developments in antitumor activity and cardiotoxicity. Pharmacol Rev 2004 56:185-229.

7 El-Kareh AW, Secomb TW: Mathematical model for comparison of bolus injection, continuous infusion, and liposomal delivery of doxorubicin to tumor cells. Neoplasia 2000;2: 325-338.

8 Lipshultz SE, Miller TL, Lipsitz SR, et al: Continuous versus bolus infusion of doxorubicin in children with ALL: long-term cardiac outcomes. Pediatrics 2012;130:1003-1011.

9 Doroshow JH, Synold TW, Somlo G, et al: Oxidative DNA base modifications in peripheral blood mononuclear cells of patients treated with high-dose infusional doxorubicin. Blood 2001;97:2839-2845.

10 Salvatorelli E, Menna P, Minotti G: Pharmacology of cardio-oncology; in Yeh ET, Ewer MS (eds): Cancer and the Heart, 2017 (in press).

11 Seymour LW: Passive tumor targeting of soluble macromolecules and drug conjugates. Crit Rev Ther Drug Carrier Syst 1992;9:135-187.

12 Menna P, Salvatorelli E, Minotti G: Cardiotoxicity of antitumor drugs. Chem Res Toxicol 2008;15:1179-1189.

13 Drummond DC, Meyer O, Hong K, et al: Optimizing liposomes for delivery of chemotherapeutic agents to solid tumors. Pharmacol Rev 1999;51:692-743.
14 Mayer LD, Dougherty G, Harasym TO, et al: The role of tumor associated macrophages in the delivery of liposomal doxorubicin to solid murine fibrosarcoma tumors. J Pharmacol Exp Ther 1997;280:1406-1414.

15 van Dalen EC, Michiels, EM, Caron HN, et al Different anthracycline derivates for reducing cardiotoxicity in cancer patients. Cochrane Database Syst Rev 2010;12:CD005006.

16 Cortes J, Di Cosimo S, Climent MA: Nonpegylated liposomal doxorubicin (TLC-D99), paclitaxel, and trastuzumab in HER-2-overexpressing breast cancer: a multicenter phase I/II study. Clin Cancer Res 2009;5:307-314.

17 Rayson D, Suter TM, Jackisch C, et al: Cardiac safety of adjuvant pegylated liposomal doxorubicin with concurrent trastuzumab: a randomized phase II trial. Ann Oncol 2012;23: 1780-1788.

18 Doroshow JH: Anthracycline antibiotic-stimulated superoxide, hydrogen peroxide, and hydroxyl radical production by NADH dehydrogenase. Cancer Res 1983;43:4543-4551.

19 Gewirtz DA: A critical evaluation of the mechanisms of action proposed for the antitumor effects of the anthracycline antibiotics adriamycin and daunorubicin. Biochem Pharmacol 1999;57:727-741.

20 Minotti G, Salvatorelli E, Menna P: Pharmacological foundations of cardio-oncology. J Pharmacol Exp Ther 2010;334:2-8.

21 Boucek RJ, Olson RD, Brenner DE, et al: The major metabolite of doxorubicin is a potent inhibitor of membrane-associated ion pumps. J Biol Chem 1987;262:15851-15856.

22 Minotti G, Recalcati S, Mordente A, et al: The secondary alcohol metabolite of doxorubicin irreversibly inactivates aconitase/iron regulatory protein-1 in cytosolic fractions from human myocardium. FASEB J 1998; 12:541552.

23 Minotti G, Ronchi R, Salvatorelli E, et al: Doxorubicin irreversibly inactivates iron regulatory proteins 1 and 2 in cardiomyocytes: evidence for distinct metabolic pathways and implications for iron-mediated cardiotoxicity of antitumor therapy. Cancer Res 2001;61: 8422-8428.

24 Salvatorelli E, Guarnieri S, Menna P, et al: Defective one or two electron reduction of the anticancer anthracycline epirubicin in human heart: relative importance of vesicular sequestration and impaired efficiency of electron addition. J Biol Chem 2006;281:1099011001.

25 Ryberg M, Nielsen D, Cortese G, et al: New insight into epirubicin cardiac toxicity: competing risks analysis of 1097 breast cancer patients. J Natl Cancer Inst 2008;100:1058-1067.

26 Salvatorelli E, Menna P, Gianni L, et al: Defective taxane stimulation of epirubicinol formation in the human heart: insight into the cardiac tolerability of epirubicin-taxane chemotherapies. J Pharmacol Exp Ther 2007;320: 790-800.

27 Salvatorelli E, Menna P, Gonzalez Paz O, et al: Pharmacokinetic characterization of amrubicin cardiac safety in an ex vivo human myocardial strip model. II. Amrubicin shows metabolic advantages over doxorubicin and epirubicin. J Pharmacol Exp Ther 2012;341: 474-483.

28 Salvatorelli E, Menna P, Gonzalez Paz O, et al: The novel anthracenedione, pixantrone, lacks redox activity and inhibits doxorubicinol formation in human myocardium: insight to explain the cardiac safety of pixantrone in doxorubicin-treated patients. J Pharmacol Exp Ther 2013;344:467-478.

29 Herbrecht R, Cernohous P, Engert A, et al: Comparison of pixantrone-based regimen (CPOP-R) with doxorubicin-based therapy (CHOP-R) for treatment of diffuse large B-cell lymphoma. Ann Oncol 2013;24:2618-2623.

30 Pettengell R, Coiffier B, Narayanan G, et al: Pixantrone dimaleate versus other chemotherapeutic agents as a single-agent salvage treatment in patients with relapsed or refractory aggressive non-Hodgkin lymphoma: a phase 3, multicentre, open-label, randomised trial. Lancet Oncol 2012;13:696-706.

31 Beeharry N, Di Rora AG, Smith MR, et al: Pixantrone induces cell death through mitotic perturbations and subsequent aberrant cell divisions. Cancer Biol Ther 2015;16:13971406.

32 Menna P, Salvatorelli E, Minotti G: Rethinking drugs from chemistry to therapeutic opportunities: pixantrone beyond anthracyclines. Chem Res Toxicol 2016;29:1270-1278.

33 Legha SS, Wang YM, Mackay B, et al: Clinical and pharmacologic investigation of the effects of a-tocopherol on adriamycin cardiotoxicity. Ann NY Acad Sci 1982;393:411-418. 
34 Myers C, Bonow R, Palmieri S, et al: A randomized controlled trial assessing the prevention of doxorubicin cardiomyopathy by $\mathrm{N}$ acetylcysteine. Semin Oncol 1983;10:53-55.

35 Minotti G, Cairo G, Monti E: Role of iron in anthracycline cardiotoxicity: new tunes for an old song? FASEB J 1999;13:199-212.

36 Doroshow JH: Role of reactive-oxygen metabolism in the cardiac toxicity of the anthracycline antibiotics; in Priebe W (ed): Anthracycline Antibiotics: New Analogues, Methods of Delivery, and Mechanisms of Action. Washington, American Chemical Society, 1995, pp 259-267.

37 Zhang S, Liu X, Bawa-Khalfe T, et al: Identification of the molecular basis of doxorubicininduced cardiotoxicity. Nat Med 2012;18: 1639-1642.

38 Lyu YL, Kerrigan JE, Lin CP, et al: Topoisomerase IIbeta mediated DNA double-strand breaks: implications in doxorubicin cardiotoxicity and prevention by dexrazoxane. Cancer Res 2007;67:8839-8846.

39 Martin E, Thougaard AV, Grauslund M, et al Evaluation of the topoisomerase II-inactive bisdioxopiperazine ICRF-161 as a protectant against doxorubicin-induced cardiomyopathy. Toxicology 2009;255:72-79.

40 Sterba M, Popelová O, Simunek T, et al: Cardioprotective effects of a novel iron chelator, pyridoxal 2-chlorobenzoyl hydrazone, in the rabbit model of daunorubicin-induced cardiotoxicity. J Pharmacol Exp Ther 2006;319: 1336-1347.

41 Popelová O, Sterba M, Simůnek T, et al: Deferiprone does not protect against chronic anthracycline cardiotoxicity in vivo. J Pharmacol Exp Ther 2008;326:259-269.

42 Speyer JL, Green MD, Zeleniuch-Jacquotte A et al: ICRF-187 permits longer treatment with doxorubicin in women with breast cancer. J Clin Oncol 1992;10:117-127.

43 Swain SM, Whaley FS, Gerber MC, et al: Cardioprotection with dexrazoxane for doxorubicin-containing therapy in advanced breast cancer. J Clin Oncol 1997;15:1318-1332.

44 Hasinoff BB, Creighton AM, Kozlowska H, et al: Mitindomide is a catalytic inhibitor of DNA topoisomerase II that acts at the bisdioxopiperazine binding site. Mol Pharmacol 1997;52:839-845.

45 Swain SM, Vici P: The current and future role of dexrazoxane as a cardioprotectant in anthracycline treatment: expert panel review. J Cancer Res Clin Oncol 2004;130:1-7.

46 Schuchter LM, Hensley ML, Meropol NJ, et al: 2002 update of recommendations for the use of chemotherapy and radiotherapy protectants: clinical practice guidelines of the American Society of Clinical Oncology. J Clin Oncol 2002;20:2895-2903.

47 Tebbi CK, London WB, Friedman D, et al: Dexrazoxane-associated risk for acute myeloid leukemia/myelodysplastic syndrome and other secondary malignancies in pediatric Hodgkin's disease. J Clin Oncol 2007;25:493-500.
48 Salze WL, Devidas M, Carroll WL, et al: Longterm results of the pediatric oncology group studies for childhood acute lymphoblastic leukemia. Leukemia 2010;24:355-370.

49 Vrooman LM, Neuberg DS, Stevenson KE, et al: The low incidence of secondary acute myelogenous leukaemia in children and adolescents treated with dexrazoxane for acute lymphoblastic leukaemia. Eur J Cancer 2011;47: 1373-1379.

50 Asselin BL, Devidas M, Chen L, et al: Cardioprotection and safety of dexrazoxane in patients treated for newly diagnosed T-cell acute lymphoblastic leukemia or advanced-stage lymphoblastic non-Hodgkin lymphoma: a report of the Children's Oncology Group Randomized Trial Pediatric Oncology Group 9404. J Clin Oncol 2016;34:854-862

51 Lipshultz SE, Franco VI, Sallan SE, et al: Dexrazoxane for reducing anthracycline-related cardiotoxicity in children with cancer: an update of the evidence. Progr Pediatr Cardiol 2014;36:39-49.

52 Limat S, Demesmay K, Voillat L, et al: Early cardiotoxicity of the $\mathrm{CHOP}$ regimen in aggressive non-Hodgkin's lymphoma. Ann Oncol 2003; 14:277-281.

53 Yang SC, Chuang MH, Li DK: The development of congestive heart failure and ventricular tachycardia after first exposure to idarubicin in a patient with acute myeloid leukaemia. Br J Clin Pharmacol 2010;69:209-211.

54 Forrest GL, Gonzalez B, Tseng W, et al: Human carbonyl reductase overexpression in the heart advances the development of doxorubicin induced cardiotoxicity in transgenic mice. Cancer Res 2000;60:5158-5164.

55 Olson LE, Bedja D, Alvey SJ, et al: Protection from doxorubicin-induced cardiac toxicity in mice with a null allele of carbonyl reductase 1 . Cancer Res 2003;63:6602-6606.

56 Blanco JG, Sun CL, Landier W, et al: Anthracycline-related cardiomyopathy after childhood cancer: role of polymorphisms in carbonyl reductase genes: a report from the Children's Oncology Group. J Clin Oncol 2012;30: 1415-1421.

57 Kalabus JL, Sanborn CC, Jamil RG, Cheng Q, Blanco JG: Expression of the anthracyclinemetabolizing enzyme carbonyl reductase 1 in hearts from donors with Down syndrome. Drug Metab Dispos 2010;38:2096-3009.

58 Hefti E, Blanco JG: Anthracycline-related cardiotoxicity in patients with acute myeloid leukemia and Down syndrome: a literature review. Cardiovasc Toxicol 2016;16:5-13.

59 Mordente A, Minotti G, Martorana GE, Silvestrini A, Giardina B, Meucci E: Anthracycline secondary alcohol metabolite formation in human or rabbit heart: biochemical aspects and pharmacologic implications. Biochem Pharmacol 2003;66:989-998.

60 Vejpongsa P, Massey MR, Acholonu SA, et al: Topoisomerase $2 \mathrm{~b}$ expression in peripheral blood predicts susceptibility to anthracyclineinduced cardiomyopathy. Circulation 2013; 128:A11619.
61 Aminkeng F, Bhavsar AP, Visscher $\mathrm{H}$, et al: A coding variant in RARG confers susceptibility to anthracycline-induced cardiotoxicity in childhood cancer. Nat Genet 2015;47:10791084.

62 Wojnowski L, Kulle B, Schirmer M, et al: $\mathrm{NAD}(\mathrm{P}) \mathrm{H}$ oxidase and multidrug resistance protein genetic polymorphisms are associated with doxorubicin-induced cardiotoxicity. Circulation 2005;112:3754-3762.

63 Reichwagen A, Ziepert M, Kreuz M, et al: Association of NADPH oxidase polymorphisms with anthracycline-induced cardiotoxicity in the RICOVER-60 trial of patients with aggressive CD20(+) B-cell lymphoma. Pharmacogenomics 2015;16:361-372.

64 Wang X, Liu W, Sun CL, et al: Hyaluronan synthase 3 variant and anthracycline-related cardiomyopathy: a report from the Children's Oncology Group. J Clin Oncol 2014;32:647653.

65 Kalay N, Basar E, Ozdogru I, et al: Protective effects of carvedilol against anthracycline-induced cardiomyopathy. J Am Coll Cardiol 2006;48:2258-2262.

66 Kaya MG, Ozkan M, Gunebakmaz O, et al: Protective effects of nebivolol against anthracycline induced cardiomyopathy: a randomized control study. Int J Cardiol 2013;167: 2306-2310.

67 Bosch X, Sitges M, Rovira M, et al: Enalapril and carvedilol for preventing chemotherapyinduced left ventricular systolic dysfunction in patients with malignant hemopathies: the OVERCOME trial. J Am Coll Cardiol 2013; 61:2355-2362

68 Georgakopoulos P, Roussou P, Matsakas E, et al: Cardioprotective effect of metoprolol and enalapril in doxorubicin-treated lymphoma patients: a prospective, parallel-group, randomized, controlled study with 36-month follow-up. Am J Hematol 2010;85:894-896.

69 Gulati G, Heck SL, Ree AH, et al: Prevention of cardiac dysfunction during adjuvant breast cancer therapy (PRADA): a $2 \times 2$ factorial, randomized, placebo-controlled, doubleblind clinical trial of candesartan and metoprolol. Eur Heart J 2016;37:1671-1680.

70 Seicean S, Seicean A, Plana JC, et al: Effect of statin therapy on the risk for incident heart failure in patients with breast cancer receiving anthracycline chemotherapy: an observational clinical cohort study. J Am Coll Cardiol 2012;60:2384-2390.

71 Schmidinger M, Zielinski CC, Vogl UM, et al: Cardiac toxicity of sunitinib and sorafenib in patients with metastatic renal cell carcinoma. J Clin Oncol 2008;26:5204-5212.

72 Zhao Z, Cui W, Zhang H, et al: Pre-treatment of a single high-dose of atorvastatin provided cardioprotection in different ischaemia/reperfusion models via activating mitochondrial KATP channel. Eur J Pharmacol 2015;751: 89-98. 